\title{
Conjugated Chromophore Arrays with Unusually Large Hole Polaron Delocalization Lengths
}

\author{
Kimihiro Susumu, ${ }^{\dagger}$ Paul R. Frail, ${ }^{\dagger}$ Paul J. Angiolillo, ${ }^{* *}$ Michael J. Therien ${ }^{\dagger *}$ \\ ${ }^{\dagger}$ Department of Chemistry, University of Pennsylvania, Philadelphia, \\ Pennsylvania 19104-6323; Department of Physics, ${ }^{*}$ St. Joseph's University, \\ Philadelphia, Pennsylvania 19131-1395
}

\section{Experimental Section}

Materials. All manipulations were carried out under nitrogen or argon previously passed through an $\mathrm{O}_{2}$ scrubbing tower (Schweitzerhall R3-11 catalyst) and a drying tower (Linde 3- $\AA$ molecular sieves) unless otherwise stated. Air sensitive solids were handled in a Braun 150-M glove box. Standard Schlenk techniques were employed to manipulate air-sensitive solutions. Methylene Chloride $\left(\mathrm{CH}_{2} \mathrm{Cl}_{2}\right)$ and tetrahydrofuran (THF) were distilled from $\mathrm{CaH}_{2}$ and $\mathrm{K} / 4$ benzoylbiphenyl, respectively, under $\mathrm{N}_{2}$. All NMR solvents were used as received. $\mathrm{ZnCl}_{2}$ was dried by heating under vacuum and stored under $\mathrm{N}_{2}$. The catalysts $\mathrm{Pd}\left(\mathrm{PPh}_{3}\right)_{4}, \mathrm{PdCl}_{2}\left(\mathrm{PPh}_{3}\right)_{2}$, and tris(dibenzylideneacetone)dipalladium $(0)\left(\mathrm{Pd}_{2} \mathrm{dba}_{3}\right)$, as well as triphenylarsine $\left(\mathrm{AsPh}_{3}\right)$ were purchased from Strem Chemicals and used as received.

Chemical shifts for ${ }^{1} \mathrm{H}$ NMR spectra are relative to tetramethylsilane (TMS) signal in the deuterated solvent (TMS, $\mathrm{d}=0.00 \mathrm{ppm})$. All $J$ values are reported in Hertz. Flash and size exclusion column chromatography was performed on the bench top, using respectively silica gel (EM Science, 230-400 mesh) and Bio-Rad Bio-Beads SX-1 as media. Mass spectra were acquired at the Mass Spectrometry Center at the University of Pennsylvania. MALDI-TOF mass spectroscopic data were obtained with a Perspective Voyager DE instrument in the Laboratory of 
Dr. Bill Degrado (Department of Biophysics, University of Pennsylvania). Samples were prepared as micromolar solutions in THF, and dithranol (Aldrich) and a-cyano-4-hydroxy cinnamic acid (Aldrich) was utilized as the matrix.

Instrumentation. Electronic spectra were recorded on an OLIS UV/vis/near-IR spectrophotometry system that is based on the optics of a Cary 14 spectrophotometer or a Shimadzu PharmSpec Uv-1700. NMR spectra were recorded on either $250 \mathrm{MHz}$ AC-250, or $500 \mathrm{MHz}$ AMX-500 Brüker spectrometers. Electron spin resonance spectroscopy was performed on the monocationic states of compounds on a Varian E-104 (77-298 K) and on a Bruker ESP 300 (4-77 K). On the Varian, spectra were acquired using an analog-to-digital converter from Scientific Software Services (EWWIN 4.21, Plymouth, MI) and frequency was measured using an EIP Autohet frequency counter (Model 351D). Temperature was measures using a thermocouple and is accurate to $+/-0.1{ }^{\circ} \mathrm{C}$. On the Bruker instrument, microwave frequency was determined using a Hewlett-Packard frequency counter (Model 5350B) and temperature was controlled using an Oxford ESR 900 continuous flow liquid helium cryostat regulated (+/- $1 \mathrm{~K}$ over the temperature range studied) with an Oxford ITC4 temperature controller. g-values were calculated using the frequency from the frequency counter and using both DPPH and weak pitch as a g-value standards to calibrate field position. Spectral simulations were performed using SimFonia (Bruker v. 1.0).

\section{General ESR Sample Preparation:}

A known amount of porphyrin was dissolved in a Schlenk tube with freshly distilled $\mathrm{CH}_{2} \mathrm{Cl}_{2}$ (2\% THF: $\mathrm{CH}_{2} \mathrm{Cl}_{2}$ was used for $\mathrm{PZn} 7$ ) such that the final concentration would be in the 
range of 100-500 $\mu \mathrm{M}$. (tris(p-bromophenyl)amminium hexachloroantimonate) was weighed out in a glovebox and charged into a Schlenk tube. Distilled and degassed $\mathrm{CH}_{2} \mathrm{Cl}_{2}$ was transferred into the oxidant's Schlenk tube and allowed to stir at room temperature for $30 \mathrm{~min}$. An aliquout was taken from the porphyrin stock solution and transferred to an ESR adapted Schlenk tube. 0.8-0.9 equivalents of oxidant was than added to the ESR tube via syringe and after mixing under Argon the tube was quickly placed into a $-78^{\circ} \mathrm{C}$ bath. Total volume in the ESR tube ranged between $0.25-0.50 \mathrm{~mL}$. Samples were measured within 30 min of mixing porphyrin with oxidant.

\section{Synthetic Procedures:}

Previously made compounds see supplemental of J. Am. Chem. Soc. 2002 124(29); 85508552.

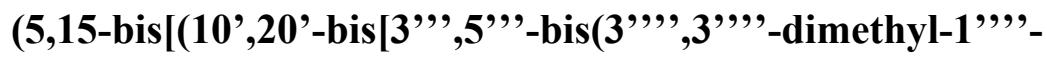

\section{butyloxy)phenyl]porphinato)zinc(II)ethyn-5'-yl]-10,20-bis[3',5'-bis(3',3',-dimethyl-1"'-} butyloxy)phenyl]porphinato)zinc(II) $\mathbf{P Z n}_{3}$ (1):

5-Ethynyl-(10,20-bis[3',5'-bis(3'”,3'-dimethyl-1''-butyloxy)phenyl]porphinato)zinc(II) (248 mg, $\left.2.61 \times 10^{-4} \mathrm{~mol}\right)$ and (5,15-dibromo-10,20-bis[3',5'-bis(3',3'”-dimethyl-1"'butyloxy)phenyl]porphinato)zinc(II) (135 $\left.\mathrm{mg}, 1.24 \times 10^{-4} \mathrm{~mol}\right)$ were charged into a Schlenk Flask with $\mathrm{AsPh}_{3}\left(49 \mathrm{mg}, 1.45 \times 10^{-4} \mathrm{~mol}\right)$ and $\mathrm{Pd}_{2} \mathrm{dba}_{3}\left(17 \mathrm{mg}, 1.86 \times 10^{-5} \mathrm{~mol}\right)$. THF:TEA (9:1) solvent mixture was degassed with an Ar purge for 30 min prior to solvent transfer. Once solvent was transferred, the reaction mixture, was stirred at $60{ }^{\circ} \mathrm{C}$ overnight under Ar. The reaction mixture was poured down a silica plug to removed catalyst and ligand. The solvent was 
removed by vacuum and the crude reaction mixture subjected to gravimetric size exclusion chromatography (BioRad SX1) using THF as the eluent. The first band was collected, solvent stripped, and then purified by silica gel chromatography using $\mathrm{CHCl}_{3}$. Yield $=266 \mathrm{mg}(77 \%$ based on dibromo starting material). ${ }^{1} \mathrm{H}$ NMR (250 MHz, $\left.\mathrm{CDCl}_{3}\right)$ : $\delta 10.45(\mathrm{~d}, 4 \mathrm{H}, \mathrm{J}=4.50 \mathrm{~Hz})$, $\delta 10.38(\mathrm{~d}, 4 \mathrm{H}, \mathrm{J}=4.68 \mathrm{~Hz}), \delta 10.08(\mathrm{~s}, 2 \mathrm{H}), \delta 9.27(\mathrm{~d}, 4 \mathrm{H}, \mathrm{J}=4.38 \mathrm{~Hz}), \delta 9.17(\mathrm{~d}, 4 \mathrm{H}, \mathrm{J}=$ $4.50 \mathrm{~Hz}), \delta 9.09$ ( d, 4H, J = 4.45Hz), $\delta 7.49$ ( d, 4H, J =2.10Hz), $\delta 7.45$ ( d, $8 \mathrm{H}, \mathrm{J}=2.23 \mathrm{~Hz}), \delta$ $6.91(\mathrm{~m}, 6 \mathrm{H}), \delta 4.24(\mathrm{~m}, 24 \mathrm{H}), \delta 1.87$ ( $\mathrm{m}, 24 \mathrm{H}), \delta 1.00$ ( $\mathrm{s}, 108 \mathrm{H})$. Vis (THF): 410, 490, 770 nm. MALDI-TOF MS m/z: $2821.2821\left(\mathrm{M}^{+}\right)$(calcd for 2823.6716).

\section{1,2-Bis[(15-bromo-10,20-bis[3',5'-bis(3',3'-dimethyl-1',-} butyloxy)phenyl]porphinato)zinc(II)-5-yl]ethyne (2):

1,2-Bis[(10,20-bis[3',5'-bis(3',,3''-dimethyl-1''-butyloxy)phenyl]porphinato)zinc(II)-5yl]ethyne (40 mg, $\left.1.42 \times 10^{-5} \mathrm{~mol}\right)$ was charged into a Schlenk flask and dissolved in $\mathrm{CHCl}_{3}:$ Pyridine $(49: 1 \mathrm{~mL})$ and cooled to $-5^{\circ} \mathrm{C}$ under Ar. N-bromosuccinimide $(6.3 \mathrm{mg}, 3.55 \mathrm{x}$ $10^{-5} \mathrm{~mol}$ ) was dissolved in $5 \mathrm{~mL}$ of $\mathrm{CHCl}_{3}$ and added to the reaction mixture drop wise while stirring. The reaction was stirred for $30 \mathrm{~min}$ and then the reaction was quenched by adding water. The organic phase was washed with $\mathrm{NHCl} 4$ (aq) 3 times, dried over $\mathrm{Na}_{2} \mathrm{SO}_{4}$, filtered, and solvent removed via vacuum. A CHCl3 silica gel column done where the first band collected was product. Yield $=20.2 \mathrm{mg}(70 \%$ based on compound 2$) .{ }^{1} \mathrm{H} \mathrm{NMR}\left(500 \mathrm{MHz}, \mathrm{CDCl}_{3}\right): \delta$ $10.34(\mathrm{~d}, 4 \mathrm{H}, \mathrm{J}=4.60 \mathrm{~Hz}), \delta 9.65(\mathrm{~d}, 4 \mathrm{H}, \mathrm{J}=4.40 \mathrm{~Hz}), \delta 9.14(\mathrm{~d}, 4 \mathrm{H}, \mathrm{J}=4.40 \mathrm{~Hz}), \delta 9.01(\mathrm{~d}$, $4 \mathrm{H}, \mathrm{J}=4.65 \mathrm{~Hz}), \delta 7.40(\mathrm{~d}, 8 \mathrm{H}, \mathrm{J}=2.30 \mathrm{~Hz}), \delta 6.90(\mathrm{t}, 4 \mathrm{H}, \mathrm{J}=2.20 \mathrm{~Hz}), \delta 4.23(\mathrm{~m}, 16 \mathrm{H}), \delta 1.86$ 
( t, 16H, J = 7.36Hz), $\delta 1.43(\mathrm{~m}, 42 \mathrm{H}), \delta 1.01(\mathrm{~s}, 72 \mathrm{H})$. Vis (THF): 410, 485, $705 \mathrm{~nm}$.

MALDI-TOF MS m/z: $2034.2104\left(\mathrm{M}^{+}\right)$(calcd for 2032.9047).

\section{1,2-Bis[(15-triisoprpylsilylethynyl-10,20-bis[3',5'-bis(3',3"'-dimethyl-1'"- butyloxy)phenyl]porphinato)zinc(II)-5-yl]ethyne (3):}

Compound $2\left(20 \mathrm{mg}, 9.9162 \times 10^{-6} \mathrm{~mol}\right)$ was charged into a Schlenk flask along with $\mathrm{PdCl}_{2}\left(\mathrm{PPh}_{3}\right)_{4}\left(1.0 \mathrm{mg}, 1.48 \times 10^{-6} \mathrm{mols}\right)$ and $\mathrm{CuI}\left(0.3 \mathrm{mg}, 1.49 \times 10^{-6} \mathrm{mols}\right)$. Solvent mixture of THF:TEA (9:1 mL) was degassed with an Ar purge for $30 \mathrm{~min}$. Sovent was transferred to the reaction flask and (triisopropylsilyl) acetylene $\left(0.1 \mathrm{~mL}, 3.9665 \times 10^{-5} \mathrm{~mol}\right)$ was added by syringe while the reaction mixture was heated to $60^{\circ} \mathrm{C}$ and stirred under Ar overnight. The reaction was quenched with water and diluted with $\mathrm{CHCl}_{3}$ and washed with $\mathrm{NH}_{4} \mathrm{Cl}$ (aq). The organic layer was collected and dried with $\mathrm{CaCl}_{2}$, filtered, and solvent removed via vacuum. The reaction mixture was purified by silica gel chromatography using $20 \%$ THF:Hexanes as the eluent. Yield $=15 \mathrm{mg}(73 \%$ based on compound 2$) .{ }^{1} \mathrm{H}$ NMR $\left(500 \mathrm{MHz}, \mathrm{CDCl}_{3}\right): \delta 10.34(\mathrm{~d}, 4 \mathrm{H}, \mathrm{J}=$ $4.60 \mathrm{~Hz}), \delta 9.65(\mathrm{~d}, 4 \mathrm{H}, \mathrm{J}=4.40 \mathrm{~Hz}), \delta 9.14(\mathrm{~d}, 4 \mathrm{H}, \mathrm{J}=4.40 \mathrm{~Hz}), \delta 9.01(\mathrm{~d}, 4 \mathrm{H}, \mathrm{J}=4.65 \mathrm{~Hz}), \delta$ $7.40(\mathrm{~d}, 8 \mathrm{H}, \mathrm{J}=2.30 \mathrm{~Hz}), \delta 6.90(\mathrm{t}, 4 \mathrm{H}, \mathrm{J}=2.20 \mathrm{~Hz}), \delta 4.23(\mathrm{~m}, 16 \mathrm{H}), \delta 1.86(\mathrm{t}, 16 \mathrm{H}, \mathrm{J}=$ 7.36Hz), $\delta 1.43(\mathrm{~m}, 42 \mathrm{H}), \delta 1.01(\mathrm{~s}, 72 \mathrm{H})$. Vis (THF): 410, 489, $710 \mathrm{~nm}$. MALDI-TOF MS m/z: $2229.1338\left(\mathrm{M}^{+}\right)$(calcd for 2231.1576).

\section{1,2-Bis[(15-ethynyl-10,20-bis[3',5'-bis(3',3'’-dimethyl-1"'- butyloxy)phenyl]porphinato)zinc(II)-5-yl]ethyne (4):}


Compound 3 (91 mg, $4.07 \times 10^{-5} \mathrm{~mol}$ ) was charged in a Schlenk Fask and dissolved in THF and cooled to $0{ }^{\circ} \mathrm{C}$ while under Ar. Tetrabutylammonium fluoride (TBAF) ( $0.85 \mathrm{~mL}, 0.1$ M TBAF solution in THF, $8.547 \times 10^{-5}$ mols) was added dropwise to the reaction mixture and allowed to stir for $15 \mathrm{~min}$ at $0{ }^{\circ} \mathrm{C}$ under Ar. At $15 \mathrm{~min}$ the reaction mixture was poured down a pre-packed $\mathrm{CHCl}_{3}$ silica gel plug and the first band was collected and solvent removed via vacuum. Yield $=77 \mathrm{mg}(98 \%$ based on compound 3$) .{ }^{1} \mathrm{H}$ NMR $\left(500 \mathrm{MHz}, \mathrm{CDCl}_{3}\right): \delta 10.34(\mathrm{~d}$, $4 \mathrm{H}, \mathrm{J}=4.60 \mathrm{~Hz}), \delta 9.65(\mathrm{~d}, 4 \mathrm{H}, \mathrm{J}=4.40 \mathrm{~Hz}), \delta 9.14(\mathrm{~d}, 4 \mathrm{H}, \mathrm{J}=4.40 \mathrm{~Hz}), \delta 9.01(\mathrm{~d}, 4 \mathrm{H}, \mathrm{J}=$ $4.65 \mathrm{~Hz}), \delta 7.40(\mathrm{~d}, 8 \mathrm{H}, \mathrm{J}=2.30 \mathrm{~Hz}), \delta 6.90(\mathrm{t}, 4 \mathrm{H}, \mathrm{J}=2.20 \mathrm{~Hz}), \delta 4.23(\mathrm{~m}, 16 \mathrm{H}), \delta 4.13(\mathrm{~s}$, $2 \mathrm{H}), \delta 1.86(\mathrm{t}, 16 \mathrm{H}, \mathrm{J}=7.36 \mathrm{~Hz}), \delta 1.01(\mathrm{~s}, 72 \mathrm{H})$. Vis (THF): 410, 489, $706 \mathrm{~nm}$. MALDI-TOF MS m/z: $1918.6101\left(\mathrm{M}^{+}\right)$(calcd for 1918.8908).

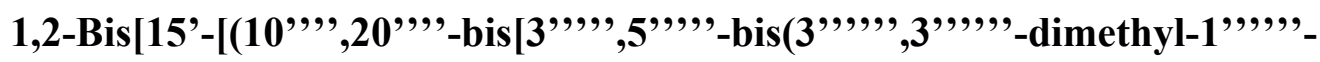

\section{butyloxy)phenyl]porphinato)zinc(II)-5,"'-ylethynyl]-10',20'-bis[3",5'-bis(3"',3'"'-} dimethyl-1"'-butyloxy)phenyl]porphinato)zinc(II)-5'-yl]ethyne $\mathrm{PZn}_{4}$ (5):

Compound 4 (32 mg, 1.66 x 10-5 mol) and (5-bromo-10,20-bis[3',5'-bis(3', 3''-dimethyl1"'-butyloxy)phenyl]porphinato)zinc(II) (35 mg, $\left.3.49 \times 10^{-5} \mathrm{~mol}\right)$ were charged in a Schlenk tube with $\mathrm{AsPh}_{3}\left(13 \mathrm{mg}, 3.98 \times 10^{-5} \mathrm{~mol}\right)$ and $\mathrm{Pd}_{2} \mathrm{dba}_{3}\left(5 \mathrm{mg}, 5.24 \times 10^{-6} \mathrm{~mol}\right)$. THF:TEA (9:1 mL) solvent mixture was degassed with an Ar purge for 30 min prior to solvent transfer. Once solvent was transferred the reaction mixture was heated to $60{ }^{\circ} \mathrm{C}$ and was stirred overnight under Ar. The following day the reaction mixture was poured down a $\mathrm{CHCl}_{3}$ column and the first band collected and the solvent stripped by vacuum. The crude reaction mixture was then chromatographed by gravimetric size exclusion (BioRad Bio-beads, SX-1) using THF as the 
eluent. First band collected and solvent stripped and another $\mathrm{CHCl}_{3}$ silica gel column was done. Yield $=43 \mathrm{mg}\left(68 \%\right.$ based on compound 4). ${ }^{1} \mathrm{H}$ NMR $\left(500 \mathrm{MHz}, \mathrm{CDCl}_{3}\right): \delta 10.46(\mathrm{~d}, 4 \mathrm{H}, \mathrm{J}=$ $4.45 \mathrm{~Hz}), \delta 10.39(\mathrm{~d}, 4 \mathrm{H}, \mathrm{J}=4.40 \mathrm{~Hz}), \delta 10.38(\mathrm{~d}, 4 \mathrm{H}, \mathrm{J}=4.65 \mathrm{~Hz}), \delta 10.08(\mathrm{~s}, 2 \mathrm{H}), \delta 9.28(\mathrm{~d}$, $4 \mathrm{H}, \mathrm{J}=4.45 \mathrm{~Hz}), \delta 9.27$ ( d, 4H, J = 4.45Hz), $\delta 9.19(\mathrm{~d}, 4 \mathrm{H}, \mathrm{J}=4.55 \mathrm{~Hz}), \delta 9.17(\mathrm{~d}, 4 \mathrm{H}, \mathrm{J}=$ $5.25 \mathrm{~Hz}), \delta 9.10(\mathrm{~d}, 4 \mathrm{H}, \mathrm{J}=4.10 \mathrm{~Hz}), \delta 7.51(\mathrm{~d}, 4 \mathrm{H}, \mathrm{J}=2.25 \mathrm{~Hz}), \delta 7.46(\mathrm{~d}, 4 \mathrm{H}, \mathrm{J}=2.15 \mathrm{~Hz}), \delta$ $6.94(\mathrm{~m}, 2 \mathrm{H}), \delta 6.92(\mathrm{~m}, 2 \mathrm{H}), \delta 4.25(\mathrm{~m}, 24 \mathrm{H}), \delta 1.88(\mathrm{~m}, 24 \mathrm{H}), \delta 1.02(\mathrm{~s}, 108 \mathrm{H})$. Vis (THF): 411, 488, 546, 604, $813 \mathrm{~nm}$. MALDI-TOF MS m/z: $3779.7911\left(\mathrm{M}^{+}\right)$(calcd for 3772.2306)

\section{(5-Triisopropylsilylethynyl-10,20-bis [3',5'-bis(3",3'-dimethyl-1"'-} butyloxy)phenyl]porphinato)zinc(II) (6):

(5-Bromo-10,20-bis[3',5'-bis(3',,3''-dimethyl-1''-butyloxy)phenyl]porphinato)zinc(II) (200 mg, $\left.1.989 \times 10^{-4} \mathrm{~mol}\right)$ was charged into Schlenk tube along with CuI $\left(6 \mathrm{mg}, 2.984 \times 10^{-4}\right.$ mol) and $\mathrm{PdCl}_{2}\left(\mathrm{PPh}_{3}\right)_{2}\left(21 \mathrm{mg}, 2.984 \times 10^{-4}\right)$. A mixture of THF:TEA $(9: 1 \mathrm{~mL})$ was degassed by constant Ar purge for 30 min and then transferred into the reaction vessel and TIPS acetylene ( $\left.0.089 \mathrm{~mL}, 3.98 \times 10^{-4} \mathrm{mols}\right)$ was then immediately added via syringe. The reaction mixture was stirred at $50{ }^{\circ} \mathrm{C}$ under Ar overnight. The following day the reaction was quenched with water and diluted with $\mathrm{CHCl}_{3}$ and washed $3 \mathrm{x}$ with $\mathrm{NH}_{4} \mathrm{Cl}$ (aq). The organic layer was collected and dried with $\mathrm{Na}_{2} \mathrm{SO}_{4}$, filtered, and solvent removed by vacuum. The purple organic residue was then chromatographed on silica gel using 20\% THF:Hexanes as the eluent. Yield $=205 \mathrm{mg}$ (93\%, based on porphyrin starting material). ${ }^{1} \mathrm{H}$ NMR $\left(250 \mathrm{MHz}, \mathrm{CDCl}_{3}\right): \delta 10.17(\mathrm{~s}, 1 \mathrm{H}), \delta$ $9.87(\mathrm{~d}, 2 \mathrm{H}, \mathrm{J}=4.48 \mathrm{~Hz}), \delta 9.33(\mathrm{~d}, 2 \mathrm{H}, \mathrm{J}=4.05 \mathrm{~Hz}), \delta 9.17(\mathrm{~d}, 2 \mathrm{H}, \mathrm{J}=3.00 \mathrm{~Hz}), \delta 9.14(\mathrm{~d}, 2 \mathrm{H}$, 
$\mathrm{J}=4.40 \mathrm{~Hz}), \delta 7.36(\mathrm{~d}, 4 \mathrm{H}, \mathrm{J}=4.60 \mathrm{~Hz}), \delta 6.86(\mathrm{~m}, 2 \mathrm{H}), \delta 4.18(\mathrm{t}, 8 \mathrm{H}, \mathrm{J}=7.29 \mathrm{~Hz}), \delta 1.85(\mathrm{~d}$,

$8 \mathrm{H}, \mathrm{J}=7.29 \mathrm{~Hz}), \delta 1.44(\mathrm{~m}, 21 \mathrm{H}), \delta 1.00(\mathrm{~s}, 36 \mathrm{H}) . \mathrm{Vis}\left(\mathrm{CHCl}_{3}\right): 428,554,594 \mathrm{~nm}$.

MALDI_TOF MS m/z: $1110.2004\left(\mathrm{M}^{+}\right)($calcd for 1106.9155).

\title{
(5-bromo-15-triisopropylsilylethynyl-10,20-bis[3',5'-bis(3",3'-dimethyl-1"'-
} butyloxy)phenyl]porphinato)zinc(II) (7):

Compound $6\left(183 \mathrm{mg}, 1.65 \times 10^{-4} \mathrm{~mol}\right)$ was dissolved in a $\mathrm{CHCl}_{3}:$ Pyridine $(49: 1 \mathrm{~mL})$ and cooled down to $-5^{\circ} \mathrm{C}$ and degassed with an Ar purge for $15 \mathrm{~min}$. N-bromosuccinimide (28 $\mathrm{mg}$, $1.65 \times 10^{-4} \mathrm{~mol}$ ) was then added and the reaction was allowed to stir for $30 \mathrm{~min}$ and then warmed to room temperature and quenched with water. The reaction mixture was then washed with $\mathrm{NaCl}$ (aq). The organic layer was collected and dried over $\mathrm{CaCl}_{2}$, filtered, and solvent removed via vacuum. The organic residue was then purified by silica gel chromatography using $20 \%$ THF:Hexanes as the eluent. Yield $=151 \mathrm{mg}\left(77 \%\right.$, based on Compound 6). ${ }^{1} \mathrm{H}$ NMR (500 $\left.\mathrm{MHz}, \mathrm{CDCl}_{3}\right): \delta 9.68(\mathrm{~d}, 2 \mathrm{H}, \mathrm{J}=4.50 \mathrm{~Hz}), \delta 9.62(\mathrm{~d}, 2 \mathrm{H}, \mathrm{J}=4.52 \mathrm{~Hz}), \delta 8.98(\mathrm{~d}, 2 \mathrm{H}, \mathrm{J}=$ $4.63 \mathrm{~Hz}), \delta 8.95(\mathrm{~d}, 2 \mathrm{H}, \mathrm{J}=4.73 \mathrm{~Hz}), \delta 7.31(\mathrm{~d}, 4 \mathrm{H}, \mathrm{J}=4.48 \mathrm{~Hz}), \delta 6.87(\mathrm{t}, 2 \mathrm{H}, \mathrm{J}=2.25 \mathrm{~Hz}), \delta$ $4.20(\mathrm{t}, 8 \mathrm{H}, \mathrm{J}=7.22 \mathrm{~Hz}), \delta 1.84(\mathrm{t}, 8 \mathrm{H}, \mathrm{J}=7.33 \mathrm{~Hz}), \delta 1.41(\mathrm{~m}, 21 \mathrm{H}), \delta 1.09(\mathrm{~s}, 36 \mathrm{H}) . \quad \mathrm{Vis}$ (THF): 432, 568, $606 \mathrm{~nm}$. MALDI-TOF MS m/z: $1186.1751\left(\mathrm{M}^{+}\right)$(calcd for 1182.4971).

\author{
(5,15-bis[(15'-triisopropylsilylethynyl-10',20'-bis[3"',5'"-bis(3,",'3,','-dimethyl-1",',- \\ butyloxy)phenyl]porphinato)zinc(II)ethyn-5'-yl]-10,20-bis[3',5'-bis(3",3"'-dimethyl-1"'- \\ butyloxy)phenyl]porphinato)zinc(II) (8):
}


Compound 7 (135 mg, $\left.1.19 \times 10^{-4} \mathrm{~mol}\right)$ and (5,15-diethynyl-10,20-bis[3',5'-bis(3',,3',dimethyl-1"'-butyloxy)phenyl]porphinato)zinc(II) (55 mg, $5.68 \times 10^{-5} \mathrm{mols}$ ) were charged into a Schlenk flask with $\mathrm{AsPh}_{3}\left(47 \mathrm{mg}, 1.43 \times 10^{-4} \mathrm{mols}\right)$ and $\mathrm{Pd}_{2} \mathrm{dba}_{3}\left(16 \mathrm{mg}, 1.70 \times 10^{-5} \mathrm{~mol}\right)$.

THF:TEA $(9: 1 \mathrm{~mL})$ solvent mixture was degassed with an Ar purge for $30 \mathrm{~min}$ and then transferred to the reaction flask. The reaction mixture was stirred at $60{ }^{\circ} \mathrm{C}$ under Ar overnight. The reaction mixture was then poured down a short silica gel column using $\mathrm{CHCl}_{3}: \mathrm{MeOH}(49: 1$ $\mathrm{mL}$ ) as the eluent. A large band was collected and the solvent stripped and then the residue was taken up in THF and put down a size exclusion column (BioRad Biobeads, SX-1) and chromatographed gravimetrically. The first band was collected and solvent removed via vacuum and the residue was purified by silica gel chromatography using $\mathrm{CHCl}_{3}: \mathrm{MeOH}(49: 1 \mathrm{~mL})$ as the eluent. Yield $=114 \mathrm{mg}(58 \%$ based on dibromo starting material $) .{ }^{1} \mathrm{H}$ NMR $\left(500 \mathrm{MHz}, \mathrm{CDCl}_{3}\right)$ : $\delta 10.35(\mathrm{~d}, 4 \mathrm{H}, \mathrm{J}=4.30 \mathrm{~Hz}), \delta 10.32(\mathrm{~d}, 4 \mathrm{H}, \mathrm{J}=4.35 \mathrm{~Hz}), \delta 9.69(\mathrm{~d}, 4 \mathrm{H}, \mathrm{J}=4.35 \mathrm{~Hz}), \delta 9.17(\mathrm{~d}$, $4 \mathrm{H}, \mathrm{J}=4.35 \mathrm{~Hz}), \delta 9.12(\mathrm{~d}, 4 \mathrm{H}, \mathrm{J}=4.35 \mathrm{~Hz}), \delta 9.00(\mathrm{~d}, 4 \mathrm{H}, \mathrm{J}=4.40 \mathrm{~Hz}), \delta 7.52(\mathrm{~d}, 4 \mathrm{H}, \mathrm{J}=$ $2.20 \mathrm{~Hz}), \delta 7.40(\mathrm{~d}, 8 \mathrm{H}, \mathrm{J}=2.30 \mathrm{~Hz}), \delta 6.99(\mathrm{~m}, 2 \mathrm{H}), \delta 6.89(\mathrm{t}, 4 \mathrm{H}, \mathrm{J}=2.05 \mathrm{~Hz}), \delta 4.23(\mathrm{~m}$, 24H), $\delta 1.81(\mathrm{~m}, 24 \mathrm{H}), \delta 1.44(\mathrm{~m}, 42 \mathrm{H}), \delta 1.03(\mathrm{~s}, 72 \mathrm{H})$. Vis (THF): 412, 490, $775 \mathrm{~nm}$. MALDI-TOF MS m/z: $3177.1150\left(\mathrm{M}^{+}\right)($calcd for 3177.5952)

\section{(5,15-bis[(15'-ethynyl-10',20'-bis[3"',5','bis(3",',3",'-dimethyl-1",',- butyloxy)phenyl]porphinato)zinc(II)ethyn-5'-yl]-10,20-bis[3',5'-bis(3",3"'-dimethyl-1"'- butyloxy)phenyl]porphinato)zinc(II) (9):}

Compound $8\left(57 \mathrm{mg}, 1.79 \times 10^{-5} \mathrm{~mol}\right)$ were dissolved in THF and cooled down to $0{ }^{\circ} \mathrm{C}$ under Ar. TBAF $\left(0.349 \mathrm{~mL}, 0.1 \mathrm{M}\right.$ TBAF in THF solution, $\left.3.49 \times 10^{-5} \mathrm{~mol}\right)$ was then added 
dropwise and the reaction mixture was allowed to stir for $15 \mathrm{~min}$ at $0{ }^{\circ} \mathrm{C}$. The reaction mixture was then directly poured down a short silica gel column using $\mathrm{CHCl}_{3}$ as the eluent. Yield $=44$ mg (84\% based on compoud 8). ${ }^{1} \mathrm{H}$ NMR $\left(500 \mathrm{MHz}, \mathrm{CDCl}_{3}\right): \delta 10.35(\mathrm{~d}, 4 \mathrm{H}, \mathrm{J}=4.30 \mathrm{~Hz}), \delta$ $10.32(\mathrm{~d}, 4 \mathrm{H}, \mathrm{J}=4.35 \mathrm{~Hz}), \delta 9.69(\mathrm{~d}, 4 \mathrm{H}, \mathrm{J}=4.35 \mathrm{~Hz}), \delta 9.17(\mathrm{~d}, 4 \mathrm{H}, \mathrm{J}=4.35 \mathrm{~Hz}), \delta 9.12(\mathrm{~d}$, $4 \mathrm{H}, \mathrm{J}=4.35 \mathrm{~Hz}), \delta 9.00(\mathrm{~d}, 4 \mathrm{H}, \mathrm{J}=4.40 \mathrm{~Hz}), \delta 7.52(\mathrm{~d}, 4 \mathrm{H}, \mathrm{J}=2.20 \mathrm{~Hz}), \delta 7.40(\mathrm{~d}, 8 \mathrm{H}, \mathrm{J}=$ 2.30Hz), $\delta 6.99(\mathrm{~m}, 2 \mathrm{H}), \delta 6.89(\mathrm{t}, 4 \mathrm{H}, \mathrm{J}=2.05 \mathrm{~Hz}), \delta 4.23(\mathrm{~m}, 24 \mathrm{H}), \delta 4.10(\mathrm{~s}, 2 \mathrm{H}), \delta 1.81($ $\mathrm{m}, 24 \mathrm{H}), \delta 1.44(\mathrm{~m}, 42 \mathrm{H}), \delta 1.03(\mathrm{~s}, 72 \mathrm{H})$. Vis (THF): 412, 490, $772 \mathrm{~nm}$. MALDI-TOF MS $\mathrm{m} / \mathrm{z}: 2864.3542\left(\mathrm{M}^{+}\right)$(calcd for 2865.3283).

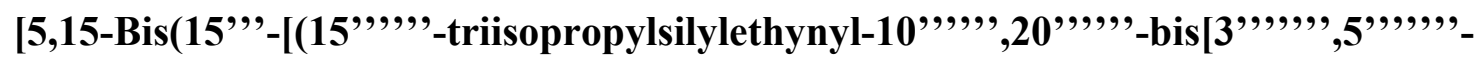
bis(3",,,,",3,",,,",-dimethyl-1",,,,"'-butyloxy)phenyl]porphinato)zinc(II)-ethyn-5,",,",yl]-

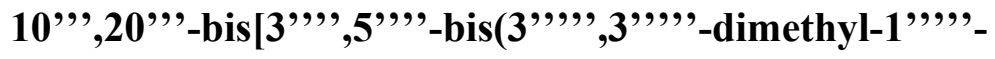

butyloxy)phenyl]porphinato)zinc(II)-ethyn-5"'-yl)-10,20-bis[3',5'-bis(3",3"'-dimethyl-1"'butyloxy)phenyl]porphinato]zinc(II) (10).

Compound 9 (73 mg, $\left.2.56 \times 10^{-5} \mathrm{~mol}\right)$ and compound 7 (64 mg, $\left.5.38 \times 10^{-5} \mathrm{~mol}\right)$ were charged into a Schlenk flask with $\mathrm{P}(\mathrm{o}-\mathrm{tolyl})_{3}\left(20 \mathrm{mg}, 6.46 \times 10^{-5} \mathrm{~mol}\right), \mathrm{CuI}\left(2 \mathrm{mg}, 8.08 \times 10^{-5}\right.$ mol), and $\mathrm{Pd}_{2} \mathrm{dba}_{3}\left(10 \mathrm{mg}, 1.08 \times 10^{-5} \mathrm{~mol}\right)$. THF:TEA $(9: 1 \mathrm{~mL})$ solvent mixture was degassed with a constant Ar purge for 30 min prior to solvent transfer. Solvent was transferred and the reaction mixture was heated to $70{ }^{\circ} \mathrm{C}$ and stirred for 2 days under Ar. The reaction mixture was poured down a short silica gel column and eluted with $\mathrm{CHCl}_{3}: \operatorname{Pyr}(99: 1 \mathrm{~mL})$. The first band was collected and solvent removed via vacuum. The residue was then taken up in THF and poured down a size exclusion column (BioRad Biobeads, SX-1, gravimetric) where the second band was 
collected and solvent removed by vacuum. Followed by a short silica gel column $\mathrm{CHCl}_{3}: \mathrm{Pyr}$ (99:1). Yield $=120 \mathrm{mg}\left(88 \%\right.$, based on compound 9). ${ }^{1} \mathrm{H}$ NMR (500 MHz, $\left.\mathrm{CDCl}_{3}\right): \delta 10.38($ $\mathrm{m}, 16 \mathrm{H}), \delta 9.69(\mathrm{~d}, 4 \mathrm{H}, \mathrm{J}=4.40 \mathrm{~Hz}), \delta 9.22(\mathrm{~m}, 4 \mathrm{H}), \delta 9.18(\mathrm{~m}, 12 \mathrm{H}), \delta 9.01(\mathrm{~d}, 4 \mathrm{H}, \mathrm{J}=$ $4.35 \mathrm{~Hz}), \delta 7.53(\mathrm{~m}, 4 \mathrm{H}), \delta 7.50(\mathrm{~d}, 4 \mathrm{H}, \mathrm{J}=2.30 \mathrm{~Hz}), \delta 7.40(\mathrm{~d}, 4 \mathrm{H}, \mathrm{J}=2.30 \mathrm{~Hz}), \delta 7.02(\mathrm{~m}$, 2H), $\delta 6.93(\mathrm{~m}, 4 \mathrm{H}), \delta 6.90(\mathrm{~m}, 4 \mathrm{H}), \delta 4.41(\mathrm{~m}, 8 \mathrm{H}), \delta 4.26(\mathrm{~m}, 40 \mathrm{H}), \delta 1.81(\mathrm{~m}, 40 \mathrm{H}), \delta 1.44$ (m, 42H), $\delta 1.03$ ( $\mathrm{s}, 180 \mathrm{H})$. Vis (THF): 431, 496, 585, $833 \mathrm{~nm}$. MALDI-TOFF MS m/z: $5072.3529\left(\mathrm{M}^{+}\right)$(calcd for 5070.4703).

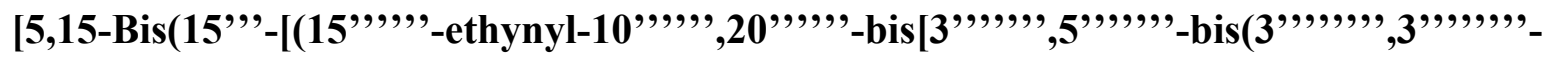
dimethyl-1",,,",'butyloxy)phenyl]porphinato)zinc(II)-ethyn-5,","'-yl]-10",,20",'bis[3",",5",'-bis(3",,",3",",-dimethyl-1",",-butyloxy)phenyl]porphinato)zinc(II)-ethyn5''-yl)-10,20-bis[3',5'-bis(3"',3"-dimethyl-1"'-butyloxy)phenyl)phenyl]porphinato]zinc(II) (11):

Compound (10) (76 mg, $1.50 \times 10^{-5}$ mols) was dissolved in THF and cooled down to $0{ }^{\circ} \mathrm{C}$ under Ar. TBAF ( $0.315 \mathrm{~mL}, 0.1 \mathrm{M}$ TBAF in THF solution, $\left.3.15 \times 10^{-5} \mathrm{~mol}\right)$ was then added dropwise and the reaction mixture was allowed to stir for $15 \mathrm{~min}$ at $0{ }^{\circ} \mathrm{C}$. The reaction mixture was then directly poured down a short silica gel column using $\mathrm{CHCl}_{3}$ as the eluent. Yield $=76$ $\mathrm{mg}\left(99 \%\right.$ based on compoud 10). ${ }^{1} \mathrm{H}$ NMR $\left(500 \mathrm{MHz}, \mathrm{CDCl}_{3}\right)$ : $\delta 10.38(\mathrm{~m}, 16 \mathrm{H}), \delta 9.69(\mathrm{~d}$, 4H, J = 4.40Hz), $\delta 9.22(\mathrm{~m}, 4 \mathrm{H}), \delta 9.18(\mathrm{~m}, 12 \mathrm{H}), \delta 9.01(\mathrm{~d}, 4 \mathrm{H}, \mathrm{J}=4.35 \mathrm{~Hz}), \delta 7.53(\mathrm{~m}, 4 \mathrm{H})$, $\delta 7.50(\mathrm{~d}, 4 \mathrm{H}, \mathrm{J}=2.30 \mathrm{~Hz}), \delta 7.40(\mathrm{~d}, 4 \mathrm{H}, \mathrm{J}=2.30 \mathrm{~Hz}), \delta 7.02(\mathrm{~m}, 2 \mathrm{H}), \delta 6.93(\mathrm{~m}, 4 \mathrm{H}), \delta 6.90($ $\mathrm{m}, 4 \mathrm{H}), \delta 4.26(\mathrm{~m}, 40 \mathrm{H}), \delta 4.13(\mathrm{~s}, 2 \mathrm{H}), \delta 1.81(\mathrm{~m}, 40 \mathrm{H}), \delta 1.44(\mathrm{~m}, 42 \mathrm{H}), \delta 1.03(\mathrm{~s}, 180 \mathrm{H})$. 
Vis (THF): 431, 496, 585, $830 \mathrm{~nm}$. MALDI-TOFF MS m/z: $4763.2853\left(\mathrm{M}^{+}\right)$(calcd for 4758.2034).

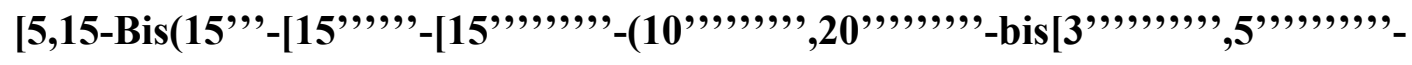

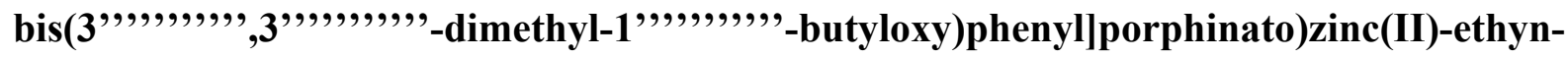

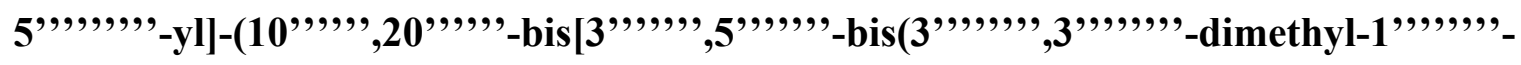

butyloxy)phenyl]porphinato)zinc(II)-ethyn-5,",,',-yl]-(10,', 20,',-bis[3,",, ,5,",'-

bis(3",,',3,",'-dimethyl-1",",'-butyloxy)phenyl]porphinato)zinc(II)-ethyn-5,"'-yl)-10,20-

bis[3',3'-dimethyl-1'-butyloxy)phenyl)phenyl]porphinato]zinc(II) $\mathbf{P Z n}_{7}(12)$ :

Compound 11 (48 mg, $\left.1.01 \times 10^{-5} \mathrm{~mol}\right)$ and (5-bromo-10,20-bis[3',5'-bis(3',,3''-

dimethyl-1"'-butyloxy)phenyl]porphinato)zinc(II) $\left(25.3 \mathrm{mg}, 2.52 \times 10^{-5} \mathrm{~mol}\right)$ were charged into a Schlenk tube with $\mathrm{Pd}_{2} \mathrm{dba}_{3}\left(5 \mathrm{mg}, 5.03 \times 10^{-6} \mathrm{~mol}\right), \mathrm{P}\left(\mathrm{o}\right.$-tolyl) $3\left(15.3 \mathrm{mg}, 5.04 \times 10^{-5} \mathrm{~mol}\right)$, and CuI (1.0 mg, $\left.5.03 \times 10^{-6} \mathrm{~mol}\right)$. THF:TEA $(9: 1 \mathrm{~mL})$ solvent mixture was degassed with constant Ar purge for $30 \mathrm{~min}$ prior to solvent transfer. Once solvent was transferred the reaction mixture was heated to $70{ }^{\circ} \mathrm{C}$ and stirred for 2 days under Ar. The reaction mixture was poured down a silica gel column eluting with $\mathrm{CHCl}_{3}: \operatorname{Pyr}(99: 1 \mathrm{~mL})$. One large band was collected and the solvent was removed via vacuum. The organic residue was then dissolved in THF and poured down a size exclusion column (BioRad Biobeads, SX-1, gravimetric). The second band was collected, solvent stripped, and further purified by silica gel chromatography using $\mathrm{CHCl}_{3}: \mathrm{Pyr}$ (99:1 $\mathrm{mL})$ as the eluent. Yield $=37 \mathrm{mg}\left(53 \%\right.$ based on compound 11). ${ }^{1} \mathrm{H} \mathrm{NMR}(500 \mathrm{MHz}$, $\left.\mathrm{CDCl}_{3}\right): \delta 10.46(\mathrm{~m}, 20 \mathrm{H}), \delta 10.21(\mathrm{~s}, 2 \mathrm{H}), \delta 9.60(\mathrm{~d}, 4 \mathrm{H}, \mathrm{J}=4.40 \mathrm{~Hz}), \delta 9.22(\mathrm{~m}, 4 \mathrm{H}), \delta 9.18($ 
$\mathrm{m}, 28 \mathrm{H}), \delta 7.53(\mathrm{~m}, 8 \mathrm{H}), \delta 7.50(\mathrm{~m}, 12 \mathrm{H}), \delta 7.40(8,8 \mathrm{H}), \delta 7.02(\mathrm{~m}, 4 \mathrm{H}), \delta 6.90(\mathrm{~m}, 10 \mathrm{H}), \delta$ $4.26(\mathrm{~m}, 56 \mathrm{H}), \delta 1.81(\mathrm{~m}, 56 \mathrm{H}), \delta 1.03(\mathrm{~m}, 252 \mathrm{H})$. Vis (THF): 418, 495, 594, $855 \mathrm{~nm}$. MALDI-TOF MS m/z: $6617.9078\left(\mathrm{M}^{+}\right)$(calcd for 6618.0060). 


\section{Figure Captions:}

Figure S1. Synthetic reaction scheme for new compounds: $\mathbf{P Z n _ { 3 }}, \mathbf{P Z n} \mathbf{n}_{4}, \mathbf{P Z n}$.

Figure S2. X-band ESR spectra of chemically prepared radical cationic states of benchmark momneric species at $298 \mathrm{~K}$. All concentrations in the range $50-100 \mu \mathrm{M}$ in $\mathrm{CH}_{2} \mathrm{Cl}_{2}$. Modulation amplitude $0.1 \mathrm{mT}$ at $100 \mathrm{kHz}$. All spectra give an envelope line width of approximately $0.6 \mathrm{mT}(6 \mathrm{G})$. All species give $14 \mathrm{~N}$ hyperfine coupling of 0.14-0.16 mT. Deatils of hyperfine couplings will be reported elsewhere.

Figure S3. X-band ESR of chemically prepared radical-cation (hole polaron state for extended structures) for monomeric, pentameric and heptameric arrays at $298 \mathrm{~K}$. Concentrations in the range of $50-100 \mu \mathrm{M}$ in $\mathrm{CH}_{2} \mathrm{Cl}_{2}$. Modulation amplitude 0.1 $\mathrm{mT}$ at $100 \mathrm{kHz}$. Microwave power 1-10 $\mathrm{mW}$ (below saturation). $\mathbf{P Z \mathbf { n } ^ { + }}$ and $\mathbf{P Z n} 5^{+}$are fitted by the first derivative of a Gaussian function and $\mathbf{P Z n} \mathbf{7}^{+}$is fit by the first derivative of a Lorentizian function (dotted line).

Figure S4. X-band simulations of an $S=1 / 2$ spin systems interacting through isotropic hyperfine coupling with ${ }^{14} \mathrm{~N}$ nuclei of spin $\mathrm{I}=1$. For the case of $\mathbf{P Z \mathbf { n } ^ { + }}, \mathrm{I}\left({ }^{14} \mathrm{~N}\right)=4$ with a hyperfine coupling of 4.2 MHz. Each subsequent simulation was performed with increasing number of interaction nuclei $\left(\mathbf{P Z n} 2^{+}, \mathrm{I}\left({ }^{14} \mathrm{~N}\right)=8\right.$; 
$\left.\mathbf{P Z n}^{3+}, I\left({ }^{14} \mathrm{~N}\right)=12 ; \mathbf{P Z n}^{5+}, \mathrm{I}\left({ }^{14} \mathrm{~N}\right)=16\right)$. In each case the isotropic hyperfine coupling constant was reduced by the number of interacting nuclei. Unresolved hyperfine couplings from nuclei other than ${ }^{14} \mathrm{~N}$ are incorporated into the line width parameter of the ${ }^{14} \mathrm{~N}$ resonance.

Figure S5. EPR spectrum of $\mathbf{P Z n 5}{ }^{+}$in $\mathrm{CH}_{2} \mathrm{Cl}_{2}(\sim 50 \mathrm{mM})$ at $4.2 \mathrm{~K}$. Microwave power, 10 mW; modulation amplitude, $0.025 \mathrm{mT}(0.25 \mathrm{G}) @ 100 \mathrm{kHz}$. Dotted line is a double Lorentzian fit with g-values of 2.0023 and 2.006. Overall linewidth approximately $0.26 \mathrm{mT}$. 
Figure S1.

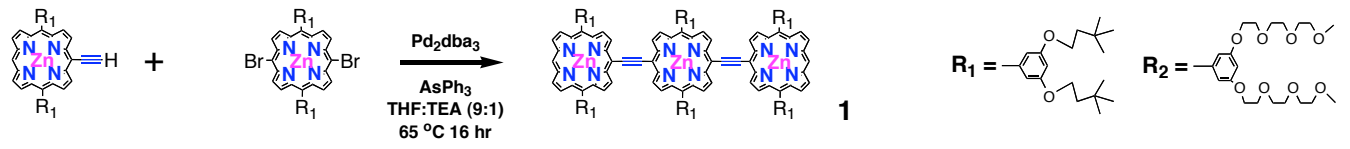
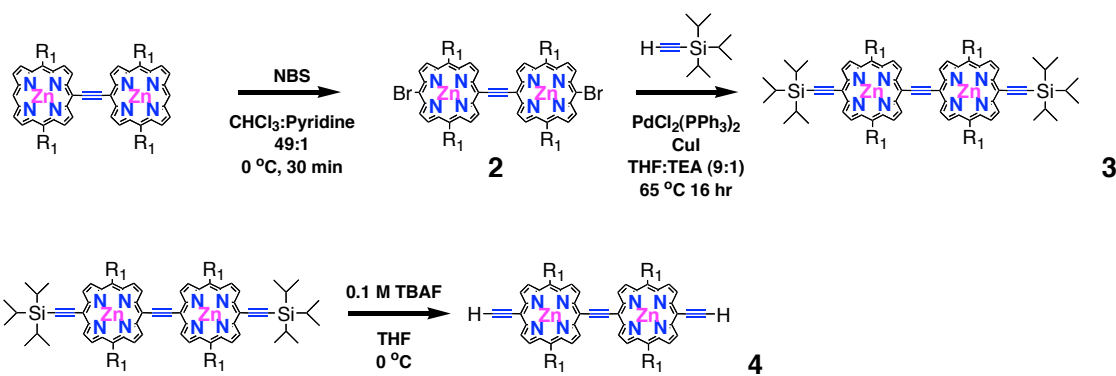

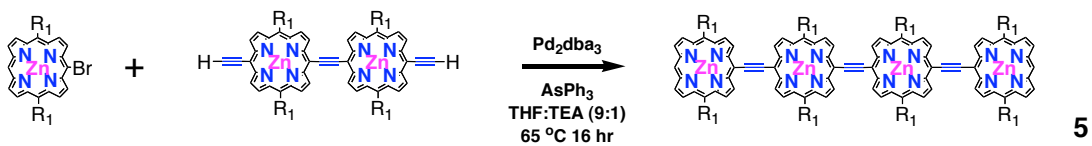

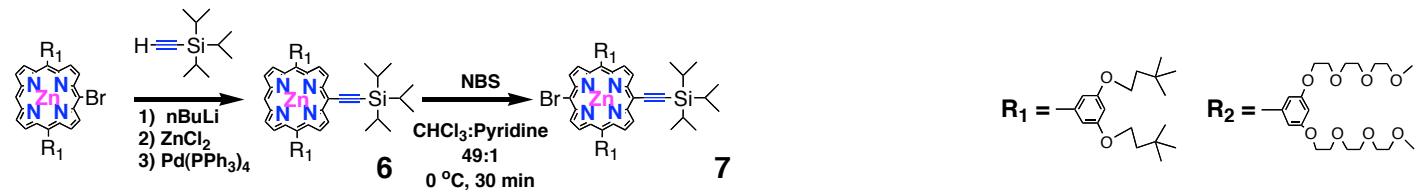

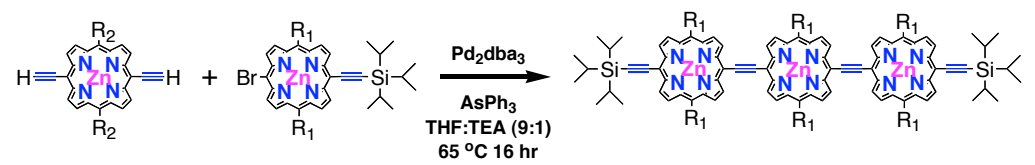

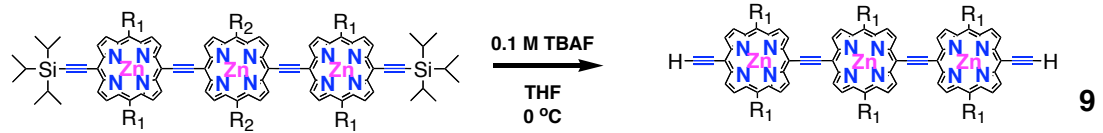
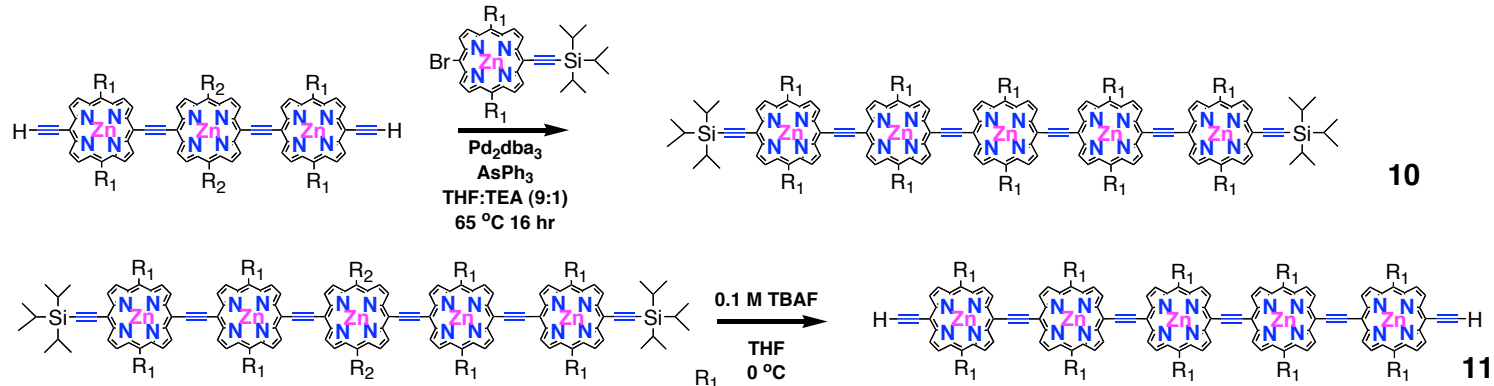

Brents

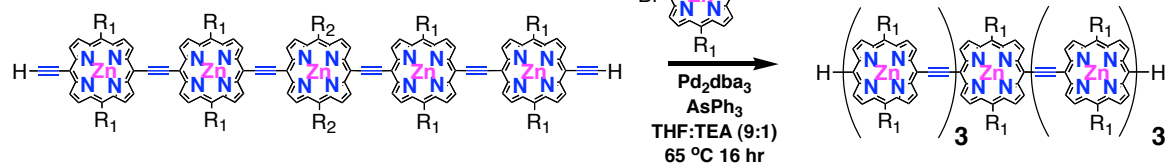


Figure S2.

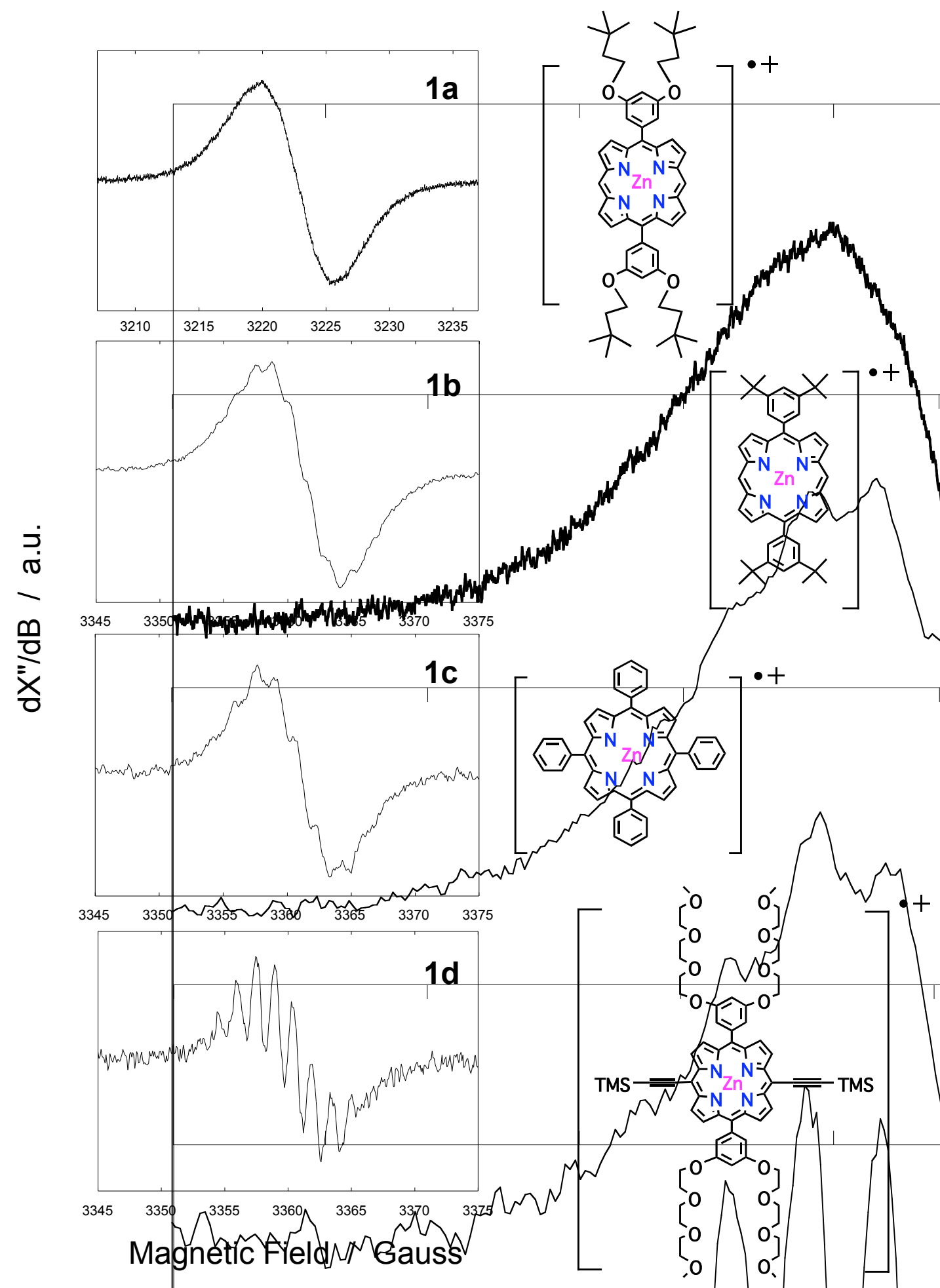


Figure S3.
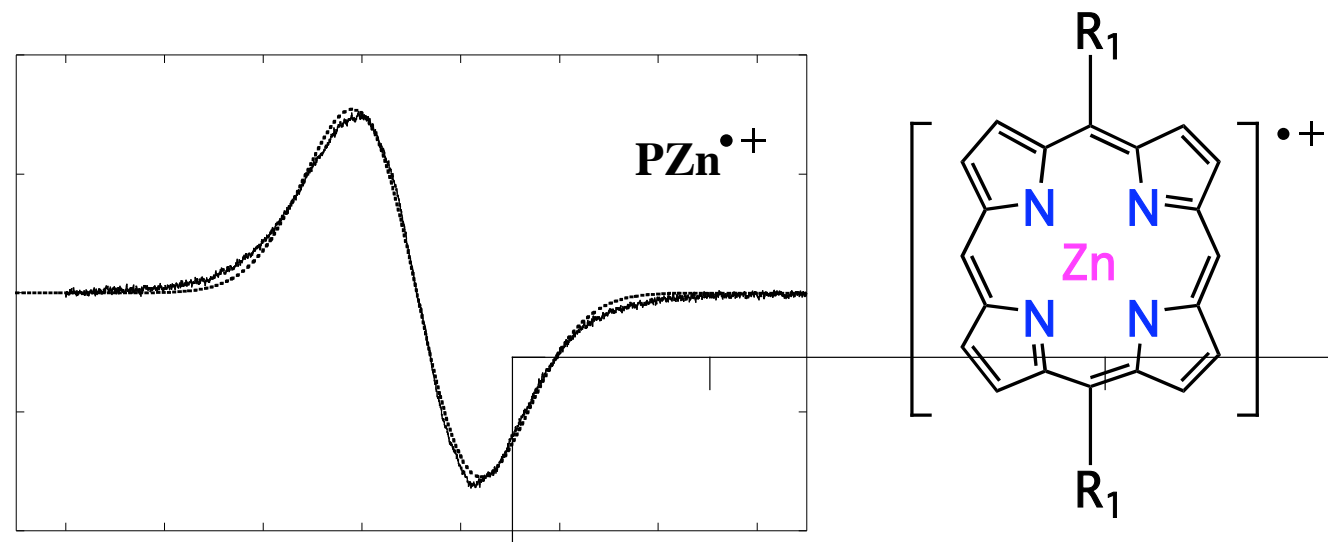

$\begin{array}{llllllll}320.5 & 321 & 321.5 & 322 & 322.5 & 323 & 323.5 & 324\end{array}$
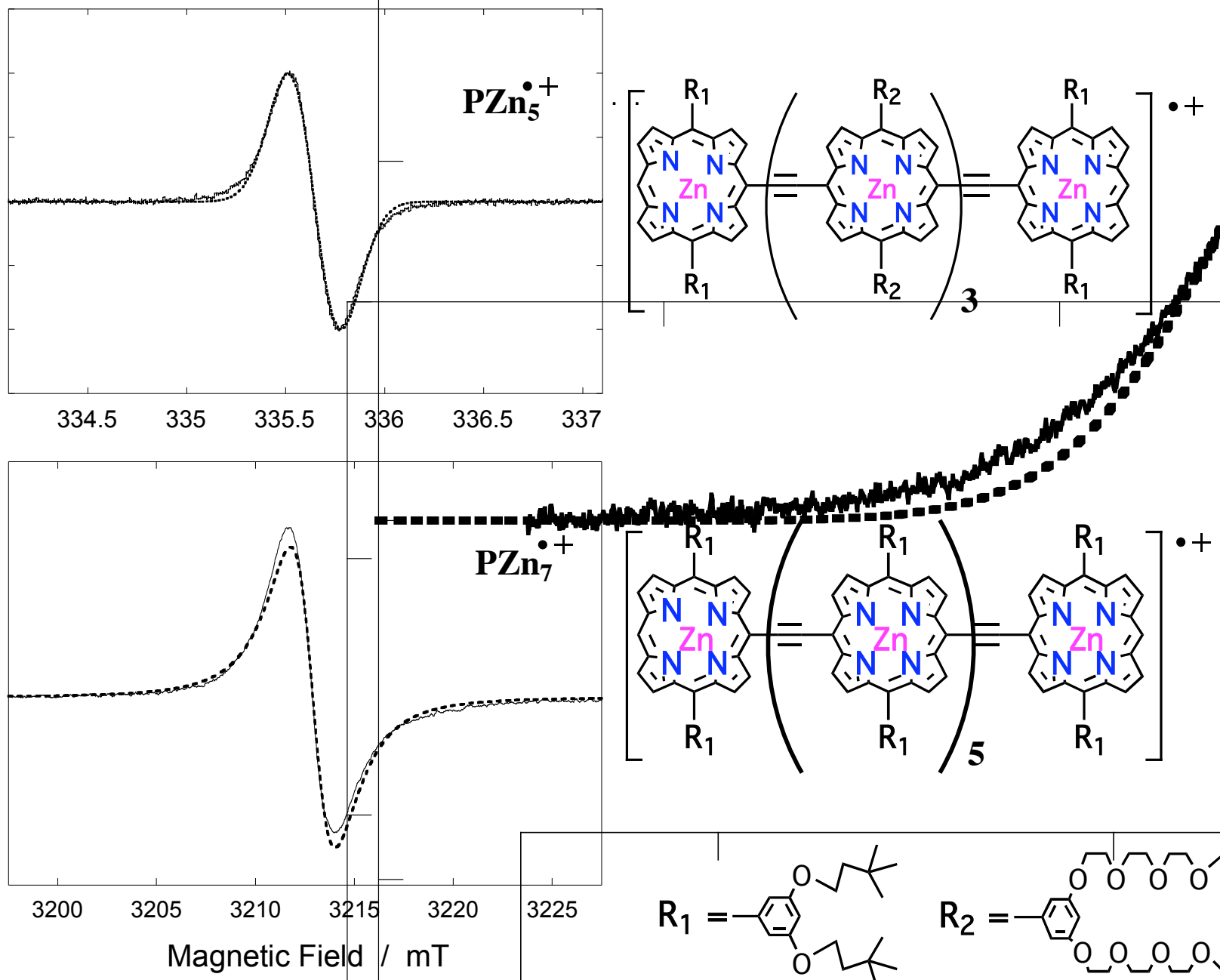

R =-4 
Figure S4.

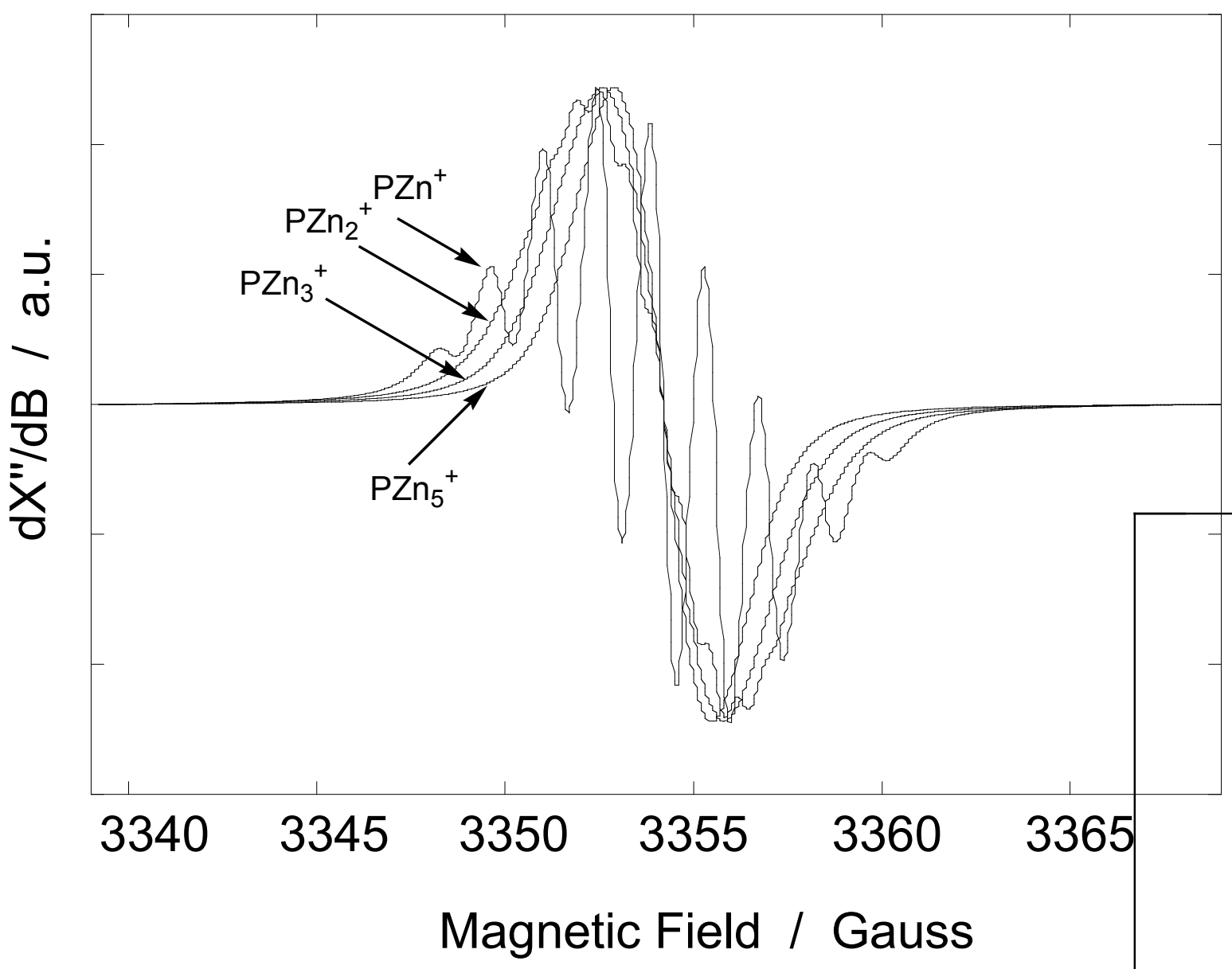


Figure S5.

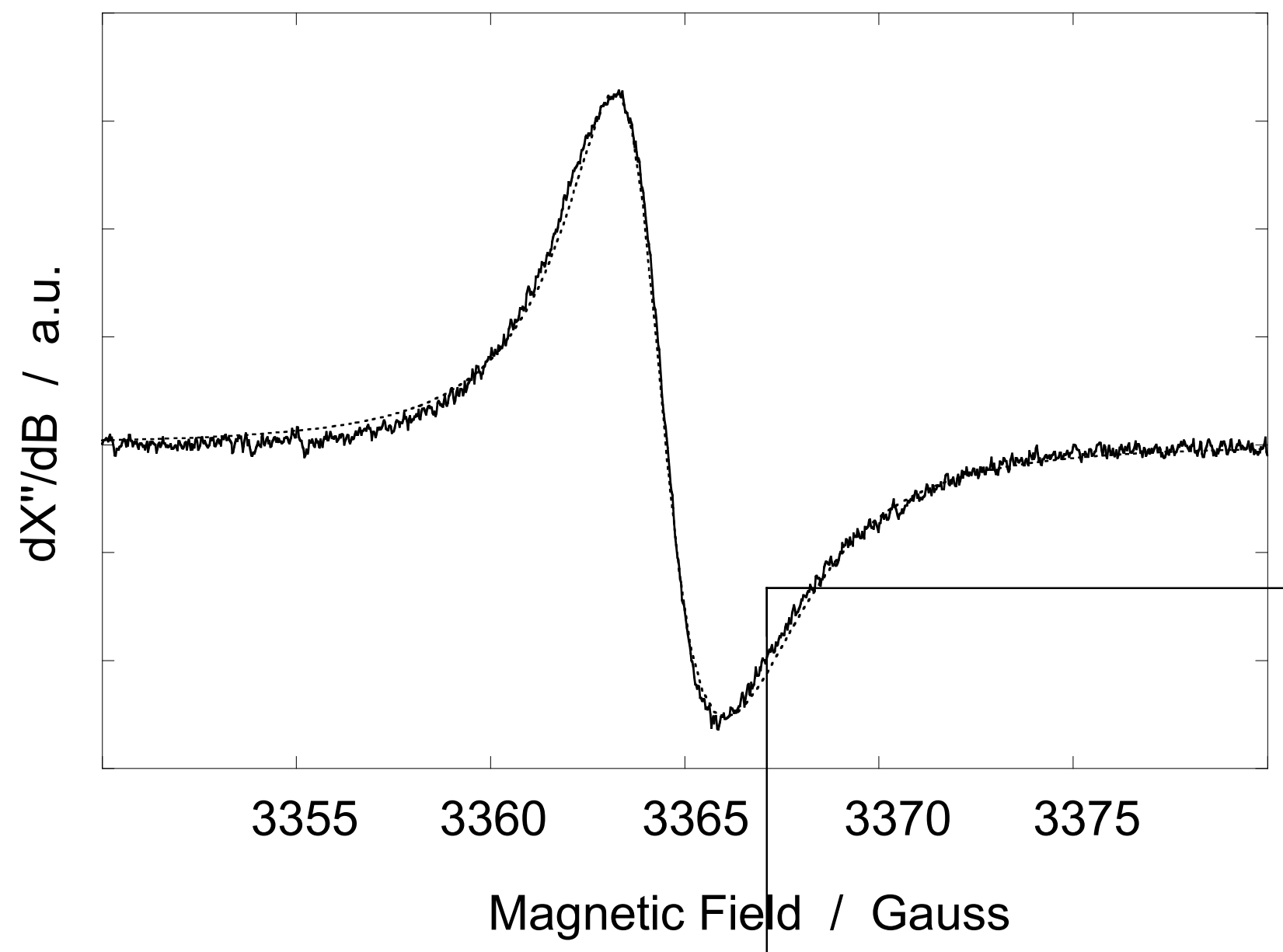

